\title{
Distribuição, fatores de risco e suscetibilidade antifúngica de espécies Candida isoladas da corrente sanguínea de pacientes críticos
}

Distribution, risk factors and antifungal susceptibility of Candida species isolated from the bloodstream of critically ill patients

Distribución, factores de riesgo y susceptibilidad antifúngica de especies de Candida aisladas del torrente sanguíneo de pacientes críticamente enfermos

\section{Resumo}

A candidemia é uma preocupação crescente em hospitais de todo o mundo, com distribuição de espécies de Candida não-albicans cada vez mais frequente em pacientes na UTI e alta taxa de mortalidade. Este estudo teve o objetivo de identificar as espécies de Candida, fatores de risco e taxas de resistência antifúngica no Estado do Rio Grande do Norte. Trata-se de um estudo transversal analítico descritivo, realizado de 2018 a 2020, com 50 pacientes internados em UTIs de três hospitais terciários, na cidade de Natal no Estado do Rio Grande do Norte. Não foram encontradas diferenças estatísticas relacionadas ao sexo, mas significância relacionada a faixa etária, principalmente nos menores de onze anos. C. albicans (48\%), C. tropicalis $(32 \%)$ e C. pelliculosa $(6 \%)$ foram as mais prevalentes. Os principais fatores de risco para candidemia, entre os pacientes dos três hospitais foi o uso de sondagem vesical (94\%), nutrição parenteral (84\%), cateter venoso central (48\%), corticoterapia (44\%) e imunossupressão (44\%). Quando comparados os casos de candidemia nos diferentes hospitais e apresentação das espécies de Candida, não houve diferença significativa na distribuição dos óbitos. Desta forma, especial atenção deve ser direcionada aqueles pacientes que apresentarem estes fatores de risco a fim de realizar uma terapia profilática prévia. O percentual de mortes foi de 16,67\% no Hospital B e $25 \%$ no Hospital C, não obtivemos dados de óbito para os pacientes do Hospital A. Observou-se resistência de $C$. albicans a anidulafungina, micafungina, voriconazol e anfotericina $\mathrm{B}$, bem como de $C$. tropicalis a estes dois últimos antifúngicos. C. pelliculosa também foi resistente ao voriconazol. Enquanto que C. parapsilosis e C. krusei apresentaram resistência à anidulafungina. A distribuição de espécies e susceptibilidade antifúngica de isolados de Candida provenientes de casos de candidemia trazem novas perspectivas ao entendimento desse grave panorama. Assim, fornece informações úteis sobre a epidemiologia, fatores de risco e padrão de susceptibilidade úteis para a seleção de agentes antifúngicos empíricos para pacientes com candidemia.

Palavras-chave: Candida albicans; Fluconazol; Resistência antifúngica; Tratamento empírico.

\begin{abstract}
Candidemia is a current concern in hospitals around the world, with a recurrent distribution of Candida species in ICU patients and a high mortality rate. This study aimed to identify Candida species, risk factors, and antifungal resistance rates in the State of Rio Grande do Norte, Brazil. This is an analytical descriptive cross-sectional study, carried out from 2018 to 2020, with 50 patients admitted to ICUs of three tertiary hospitals, in the city of Natal, State of Rio Grande do Norte, Brazil. No statistical differences were related to sex, but it was related to age group, especially in those under eleven years old. Candida albicans (48\%), C. tropicalis (32\%), and C. pelliculosa (6\%) were the most prevalent species. The main risk factor for candidemia observed in the study was the use of urinary catheters (94\%), parenteral nutrition
\end{abstract}


(84\%), a central venous catheter (48\%), corticotherapy (44\%), and immunosuppression (44\%). No significant statistical difference in the distribution of deaths when compared to the hospitals and the presentation of Candida species. Thus, special attention must be directed to patients who present these risk factors in order to undergo a previous prophylactic therapy. The percentage of deaths was $16.67 \%$ in Hospital B, 25\% in Hospital C, and 0\% for patients in Hospital A. In Candida albicans the phenomenon of antifungal resistance was related to anidulafungin, micafungin, voriconazole, and amphotericin B, as well as from $C$. tropicalis to these last two antifungals. Candida pelliculosa was also resistant to voriconazole. While $C$. parapsilosis and $C$. krusei showed resistance to anidulafungin. The species distribution and antifungal susceptibility of Candida isolated from candidemia cases bring new perspectives to the understanding of this serious scenario. Thus, it provides useful information on the epidemiology, risk factors, and susceptibility pattern useful for the selection of empirical antifungal agents for patients with candidemia.

Keywords: Candida albicans; Fluconazole; Antifungal resistance; Empirical treatment.

\section{Resumen}

La candidemia es una preocupación actual en hospitales de todo el mundo, con una distribución recurrente de especies de Candida en pacientes de UCI y una alta tasa de mortalidad. Este estudio tuvo como objetivo identificar especies de Candida, factores de riesgo y tasas de resistencia a los antifúngicos en el estado de Rio Grande do Norte, Brasil. Se trata de un estudio analítico descriptivo transversal, realizado de 2018 a 2020, con 50 pacientes ingresados en UCI de tres hospitales terciarios, en la ciudad de Natal, Estado de Rio Grande do Norte, Brasil. No hubo diferencias estadísticas relacionadas con el sexo, pero sí con el grupo de edad, especialmente en los menores de once años. Candida albicans (48\%), C. tropicalis (32\%) y C. pelliculosa (6\%) fueron las especies más prevalentes. El principal factor de riesgo de candidemia observado en el estudio fue el uso de catéteres urinarios (94\%), nutrición parenteral (84\%), catéter venoso central (48\%), corticoterapia (44\%) e inmunosupresión (44\%). No hay diferencia estadística significativa en la distribución de las muertes en comparación con los hospitales y la presentación de especies de Candida. Por tanto, se debe prestar especial atención a los pacientes que presenten estos factores de riesgo para poder someterse a una terapia profiláctica previa. El porcentaje de muertes fue del 16,67\% en el Hospital B, del 25\% en el Hospital C y del $0 \%$ para los pacientes del Hospital A. En Candida albicans el fenómeno de la resistencia antifúngica se relacionó con anidulafungina, micafungina, voriconazol y anfotericina $\mathrm{B}$, así como con desde $C$. tropicalis hasta estos dos últimos antifúngicos. Candida pelliculosa también fue resistente al voriconazol. Mientras que C. parapsilosis y C. krusei mostraron resistencia a la anidulafungina. La distribución de especies y la susceptibilidad antifúngica de Candida aislada de casos de candidemia aportan nuevas perspectivas a la comprensión de este grave escenario. Por lo tanto, proporciona información útil sobre la epidemiología, los factores de riesgo y el patrón de susceptibilidad útil para la selección de agentes antimicóticos empíricos para pacientes con candidemia.

Palabras clave: Candida albicans; Fluconazol; Resistencia a los antifúngicos; Tratamiento empírico.

\section{Introdução}

O controle das infecções por Candida é um desafio na clínica moderna (Santos et al. 2018). Como problema crescente nos hospitais em todo o mundo, a candidemia apresenta altos índices de morbidade e mortalidade, atingindo até $70 \%$ dos pacientes com esta infecção (Yapar, 2014, Li et al. 2017, Pappas et al. 2018). Na corrente sanguínea, este fungo representa o terceiro microrganismo mais isolado em hemoculturas como causa de candidemia nos estudos de base populacional (Pfaller et al. 2014, Wu et al. 2017, Doi et al. 2016).

A epidemiologia da candidemia varia geograficamente, apresentando maior risco em desenvolver infecção por Candida os pacientes internados em Unidade de Terapia Intensiva (UTI), em uso de dispositivos médicos-invasivos, antibioticoterapia de largo espectro, imunocomprometidos, crianças abaixo de 1 ano de idade e idosos (Matta; Souza; Colombo 2017, Lovero et al. 2016, Li et al. 2017, Chakrabarti et al. 2020). Embora C. albicans ainda seja a espécie considerada mais comum da candidemia, existe uma preocupação com o aumento das taxas de infecções invasivas por espécies de Candida não-albicans e resistência a antifúngicos em diferentes partes do mundo. Esse cenário necessita de controle destas infecções através do diagnóstico precoce e prevenção (Pinhati et al. 2016, Doi et al. 2016, Santos et al. 2018).

$\mathrm{Na}$ prática laboratorial, comumente o diagnóstico tem sido baseado no isolamento do fungo em cultura, como o CHROMagar Candida, usado como um meio seletivo e diferencial (Ghosh et al. 2015, Kaur and Chakrabarti 2017). Contudo, a automatização da hemocultura continua sendo a principal prática de diagnóstico da candidemia, porém ainda com baixa sensibilidade (Skrobik; Laverdiere 2013, Merseguel et al. 2015, Li et al. 2017). Nos últimos anos a identificação das espécies de Candida evoluiu de métodos convencionais baseados em bioquímicos manuais para testes moleculares e baseados em proteínas 
e ácidos nucleicos automatizados, como MALDI-TOF MS, que tem revolucionado o diagnóstico dessa infecção, com tempo resposta menor do que três horas da identificação da espécie críptica de Candida.

No cenário atual , apesar dos avanços clínicos, tem sido crescente o desenvolvimento de resistência entre espécies de Candida, principalmente em relação aos azólicos, merecendo destaque as cepas de C. glabrata, . parapsilosis e $C$. tropicalis (Santos et al. 2018, Andersen et al. 2016, Matta; Souza; Colombo 2017). Assim, o conhecimento da epidemiologia local, diagnóstico e perfil de suscetibilidade antifúngica das espécies de Candida são essenciais para orientar estratégias de prevenção e tratamento, dirimindo a carga de candidemia, morbidade e mortalidade associadas.

O presente estudo fornece um panorama das espécies, fatores de risco e taxas de resistência antifúngica entre as espécies de Candida, obtidas em amostras da corrente sanguínea de pacientes internados em Unidade de Terapia Intensiva em hospitais terciários no Estado do Rio Grande do Norte, Brasil.

\section{Métodos}

\subsection{Desenho do estudo e coleta de dados dos pacientes}

No período de abril de 2018 a fevereiro de 2020 conduzimos um estudo transversal analítico descritivo com 50 pacientes internados em UTIs de três hospitais terciários, localizados na cidade do Natal, no Estado do Rio Grande do Norte. O Laboratório de Micologia Médica do Departamento de Micologia da Universidade Federal de Pernambuco (UFPE) e a Central Analítica do Centro de Tecnologias Estratégicas do Nordeste (CETENE) foram os centros de referência para manipulação das amostras neste estudo.

As instituições participantes da pesquisa foram três hospitais públicos terciários do Estado Rio Grandes do Norte, que possuem infraestrutura para o atendimento em UTI. A seleção dos pacientes e variáveis categóricas para este estudo foi definida de acordo com a obtenção das amostras, leveduras pertencentes ao gênero Candida. Depois de selecionados os participantes, os dados clínicos, características demográficas, comorbidades subjacentes e terapêutica antifúngica, foram obtidos através dos prontuários nas diferentes instituições. A pesquisa foi aprovada pelo Comitê de Ética em Pesquisa com seres humanos, com CAAE: 81413517.4.0000.5208, sendo seguidos pelos pesquisadores, todos os preceitos éticos para o desenvolvimento do estudo.

\subsection{Identificação do microrganismo e suscetibilidade antifúngica}

As amostras foram inoculadas em meio CHROMagar Candida. A confirmação das espécies foi realizada por espectrometria de massas (MALDI-TOF Autoflex III Bruker Laser nd:yag smartbeam, Bruker Daltonics Inc., USA/Germany) e sequenciamento da região ITS.

Para confirmação taxonômica, o DNA dos isolados foi extraído segundo Inácio et al. (2016) utilizando o protocolo modificado do CTAB. Após a extração, o DNA foi ressuspendido em 50 $\mu 1$ de água MiliQ, quantificado e diluído a 10ng/ $\mu$ L. Para identificação das espécies foram realizadas reações de PCR convencional utilizado os seguintes primers ITS1 e ITS4 (White et al.,1990). Os parâmetros utilizados nas reações de PCR consistiram de desnaturação inicial a $95^{\circ} \mathrm{C}$ por 5 min, realizada em 30 ciclos de $95^{\circ} \mathrm{C}$ por $30 \mathrm{~s}, 56^{\circ} \mathrm{C}$ por $30 \mathrm{~s}, 72^{\circ} \mathrm{C}$ por $30 \mathrm{~s}$ e extensão final a $72^{\circ} \mathrm{C}$ por $10 \mathrm{~min}$. Ao final do processo, as amostras foram purificadas por meio do kit GenJET PCR Purification (Fermentas, UK) e os fragmentos de DNA genômico sequenciados na Plataforma de sequenciamento-LABCEN/CCB (UFPE) segundo protocolos preestabelecidos do laboratório parceiro.

As sequências consenso foram obtidas por meio do programa Sequencher 4.7, sendo as sequências de nucleotídeos submetidas a ferramenta BLAST do GenBank (National Center of Biotechnology Information, http://www.ncbi.nlm.nih.gov) para busca por sequências similares. As sequências obtidas nesse estudo foram alinhadas com as sequências de confiança depositadas no banco de dados e analisadas filogeneticamente com o auxílio do programa MEGA7. 
Foram realizados testes de sensibilidade antifúngica, para todas as cepas de Candida, de acordo com as diretrizes CLSI M27-A3 e M27-S4 (CLSI, 2008, 2012), para fluconazol $(0.125-64 \mu \mathrm{g} / \mathrm{mL})$, anfotericina B, voriconazol, caspofungina, micafungina e anidulafungina (todos 0,03-16 $\mu \mathrm{g} / \mathrm{mL}$ ). As concentrações inibitórias mínimas (CIMs) dos antifúngicos foram obtidas após 24 e 48 horas de incubação a $35^{\circ} \mathrm{C}$ pela pontuação de crescimento para cada uma das cepas analisadas em comparação com as cepas controle, lidos conforme instruções do protocolo CLSI M27-A3. Foram usados como controle de qualidade C. krusei ATCC 6258 e C. parapsilosis ATCC 22019. O teste de sensibilidade antifúngica foi realizado em duplicata para cada isolado.

\subsection{Análises estatísticas}

As variáveis categóricas foram expressas por meio de suas frequências e porcentagens, para análise de associação entre elas, utilizou-se o teste Qui-quadrado, considerando significante o $p$-valor $<0,05$. Dessa forma, se $\mathrm{p}$-valor for significante, rejeitase a hipótese nula e conclui-se que a hipótese de dependência entre as variáveis é verdadeira.

Foi analisada a sobrevida para óbito através da análise de Kaplan-Meier, considerando o teste de long rank para testar a hipótese de que não há diferença na distribuição da sobrevivência nos grupos avaliados, considerando significante o $p$-valor $<0,05$.

\section{Resultados}

Foram avaliados 50 pacientes dos quais 20 amostras do Hospital A (HA), 18 eram do Hospital B (HB) e 12 do Hospital C (HC). Do total de 50 pacientes, 28 (56\%) eram do sexo masculino e 20 (40\%) tinham idade entre 0 e 10 anos. Ao comparar o perfil dos três grupos de pacientes atendidos nos hospitais, em relação ao sexo e faixa etária, observou-se que não há diferença estatistica relacionada ao sexo (p-valor:0,436) e valor significante foi observado quando analisado as diferentes faixas etárias dos pacientes ( $\mathrm{p}$-valor $<0,001$ ), sobretudo naqueles menores de 11 anos (Tabela 1 ).

Em relação às espécies de Candida, foram identificadas nas culturas dos pacientes, C. albicans (n=24/50, 48\%), C. tropicalis $(\mathrm{n}=16 / 50,32 \%)$, C. pelliculosa $(\mathrm{n}=3 / 50,6 \%)$, C. parapsilosis $(\mathrm{n}=2 / 50,4 \%)$, . glabrata $(\mathrm{n}=2 / 50,4 \%)$, C. metapsilosis $(\mathrm{n}=1 / 50,2 \%), C$. orthopsilosis $(\mathrm{n}=1 / 50,2 \%)$ e $C$. krusei $(\mathrm{n}=1 / 50,2 \%)$. Não havendo diferença estatística da distribuição dessas espécies dentre os hospitais (p-valores: 0,833 e 0,169, respectivamente) (Tabela 2).

Dentre os fatores de risco nas UTIs, analisados no estudo, identificou-se maior incidência entre os pacientes dos três hospitais quanto ao uso de sondagem vesical (47 pacientes, 94\%), nutrição parenteral (42 pacientes, $84 \%$ ), cateter venoso central (24 pacientes, 48\%), corticoterapia (22 pacientes, 44\%) e imunossupressão (22 pacientes, 44\%). Ainda, foi verificado que, dentre os fatores de risco estudados, aqueles que mais estavam relacionados com o surgimento da candidemia foram o uso de antibiótico de largo espectro, corticosteróides, cirurgia prévia, cardiopatia, câncer, hipertensão, diabetes mellitus, neutropenia; quimioterapia e imunossupressão com significância estatística p < 0,05. Contudo, o uso de Cateter venoso central, ventilação mecânica, nutrição parenteral e sonda vesical não estavam associados com o surgimento de infecções por Candida. Observou-se uso maior de fluconazol no HA $(55 \%$; 11/20) e HB $(44 \%, 8 / 18)$, como também na terapia com anfotericina $(45 \%, 9 / 20)$ e $(38,9 \%, 7 / 18)$ respectivamente. Um dado interessante observado foi que, apesar de $46 \%$ os pacientes estarem em uso de fluconazol, $40 \%$ com anfotericina e 14\% com itraconazol, o uso de antifúngicos não evitou o surgimento da candidemia, com p<0,129 (Tabela 1). 
Research, Society and Development, v. 10, n. 7, e54710716731, 2021

(CC BY 4.0) | ISSN 2525-3409 | DOI: http://dx.doi.org/10.33448/rsd-v10i7.16731

Tabela1: Características clínicas e epidemiológicas dos pacientes atendidos.

\begin{tabular}{|c|c|c|}
\hline Perfil dos pacientes atendidos & Episódios & P Valor \\
\hline Sexo masculino & $28(56 \%)$ & 0,436 \\
\hline Sexo feminino & $22(44 \%)$ & \\
\hline Idade $>0$ anos & $50(100 \%)$ & $<0,001$ \\
\hline Espécies de Candida & 8 (50 isolados) & 0,169 \\
\hline Hospitais & 3 (50 episódios) & 0,833 \\
\hline Cateter Venoso Central & $24(48 \%)$ & 0,942 \\
\hline Ventilação mecânica & $22(44 \%)$ & 0,858 \\
\hline Antibiótico de largo espectro & $44(88 \%)$ & 0,006 \\
\hline Antifúngico & $50(100 \%)$ & 0,129 \\
\hline Nutrição parenteral & $42(84 \%)$ & 0,681 \\
\hline Sonda vesical & $47(94 \%)$ & 0,091 \\
\hline Corticoterapia & $22(44 \%)$ & $<0,001$ \\
\hline Cirurgia prévia & $6(12 \%)$ & 0,103 \\
\hline Hemodiálise & $2(4 \%)$ & 0,499 \\
\hline Cardiopatia & $7(14 \%)$ & 0,004 \\
\hline Neoplasia & $10(20 \%)$ & 0,012 \\
\hline Diabetes mellitus & $10(20 \%)$ & 0,01 \\
\hline Hipertensão & $13(26 \%)$ & $<0,001$ \\
\hline Neutropenia & $9(18 \%)$ & 0,004 \\
\hline Quimioterapia & $4(8 \%)$ & 0,038 \\
\hline Imunossupressão & $22(44 \%)$ & 0,001 \\
\hline
\end{tabular}

Fonte: Autores. 
Tabela 2: Número de isolados de Candida spp. identificados por fatores de risco do estudo em cada hospital.

\begin{tabular}{|c|c|c|c|c|c|c|c|c|c|c|}
\hline & \multirow{3}{*}{ Variáveis } & \multicolumn{8}{|c|}{ HOSPITAL } & \multirow{3}{*}{ p-valor* } \\
\hline & & \multicolumn{2}{|c|}{ HGT } & \multicolumn{2}{|c|}{ HIVS } & \multicolumn{2}{|c|}{ HMWG } & \multicolumn{2}{|c|}{ Total } & \\
\hline & & $\mathrm{n}$ & $\%$ & $\mathrm{n}$ & $\%$ & $\mathrm{n}$ & $\%$ & $\mathrm{n}$ & $\%$ & \\
\hline \multirow{11}{*}{ Isolados } & CRN & 0 & $0,0 \%$ & 1 & $5,0 \%$ & 0 & $0,0 \%$ & 1 & $2,0 \%$ & \multirow{11}{*}{0,833} \\
\hline & CRN1 & 5 & $27,8 \%$ & 2 & $10,0 \%$ & 4 & $33,3 \%$ & 11 & $22,0 \%$ & \\
\hline & CRN2 & 3 & $16,7 \%$ & 5 & $25,0 \%$ & 2 & $16,7 \%$ & 10 & $20,0 \%$ & \\
\hline & CRN3 & 4 & $22,2 \%$ & 4 & $20,0 \%$ & 3 & $25,0 \%$ & 11 & $22,0 \%$ & \\
\hline & CRN4 & 4 & $22,2 \%$ & 5 & $25,0 \%$ & 2 & $16,7 \%$ & 11 & $22,0 \%$ & \\
\hline & CRN5 & 1 & $5,6 \%$ & 0 & $0,0 \%$ & 1 & $8,3 \%$ & 2 & $4,0 \%$ & \\
\hline & CRN6 & 0 & $0,0 \%$ & 1 & $5,0 \%$ & 0 & $0,0 \%$ & 1 & $2,0 \%$ & \\
\hline & CRN7 & 0 & $0,0 \%$ & 1 & $5,0 \%$ & 0 & $0,0 \%$ & 1 & $2,0 \%$ & \\
\hline & CRN8 & 0 & $0,0 \%$ & 1 & $5,0 \%$ & 0 & $0,0 \%$ & 1 & $2,0 \%$ & \\
\hline & CRN9 & 1 & $5,6 \%$ & 0 & $0,0 \%$ & 0 & $0,0 \%$ & 1 & $2,0 \%$ & \\
\hline & Total & 18 & $100,0 \%$ & 20 & $100,0 \%$ & 12 & $100,0 \%$ & 50 & $100,0 \%$ & \\
\hline \multirow{9}{*}{ Espécies } & C. albicans & 6 & $33,3 \%$ & 10 & $50,0 \%$ & 8 & $66,7 \%$ & 24 & $48,0 \%$ & \multirow{9}{*}{0,169} \\
\hline & C. glabrata & 1 & $5,6 \%$ & 0 & $0,0 \%$ & 1 & $8,3 \%$ & 2 & $4,0 \%$ & \\
\hline & C. krusei & 1 & $5,6 \%$ & 0 & $0,0 \%$ & 0 & $0,0 \%$ & 1 & $2,0 \%$ & \\
\hline & C. metapsilosis & 1 & $5,6 \%$ & 0 & $0,0 \%$ & 0 & $0,0 \%$ & 1 & $2,0 \%$ & \\
\hline & C. orthopsilosis & 0 & $0,0 \%$ & 1 & $5,0 \%$ & 0 & $0,0 \%$ & 1 & $2,0 \%$ & \\
\hline & C. parapsilosis & 2 & $11,1 \%$ & 0 & $0,0 \%$ & 0 & $0,0 \%$ & 2 & $4,0 \%$ & \\
\hline & C. pelliculosa & 3 & $16,7 \%$ & 0 & $0,0 \%$ & 0 & $0,0 \%$ & 3 & $6,0 \%$ & \\
\hline & C. tropicalis & 4 & $22,2 \%$ & 9 & $45,0 \%$ & 3 & $25,0 \%$ & 16 & $32,0 \%$ & \\
\hline & Total & 18 & $100,0 \%$ & 20 & $100,0 \%$ & 12 & $100,0 \%$ & 50 & $100,0 \%$ & \\
\hline
\end{tabular}

*teste qui-quadrado

Fonte: Autores. 
Tabela 3: Taxa de mortalidade, por hospital, ocasionado pelas espécies de Candida.

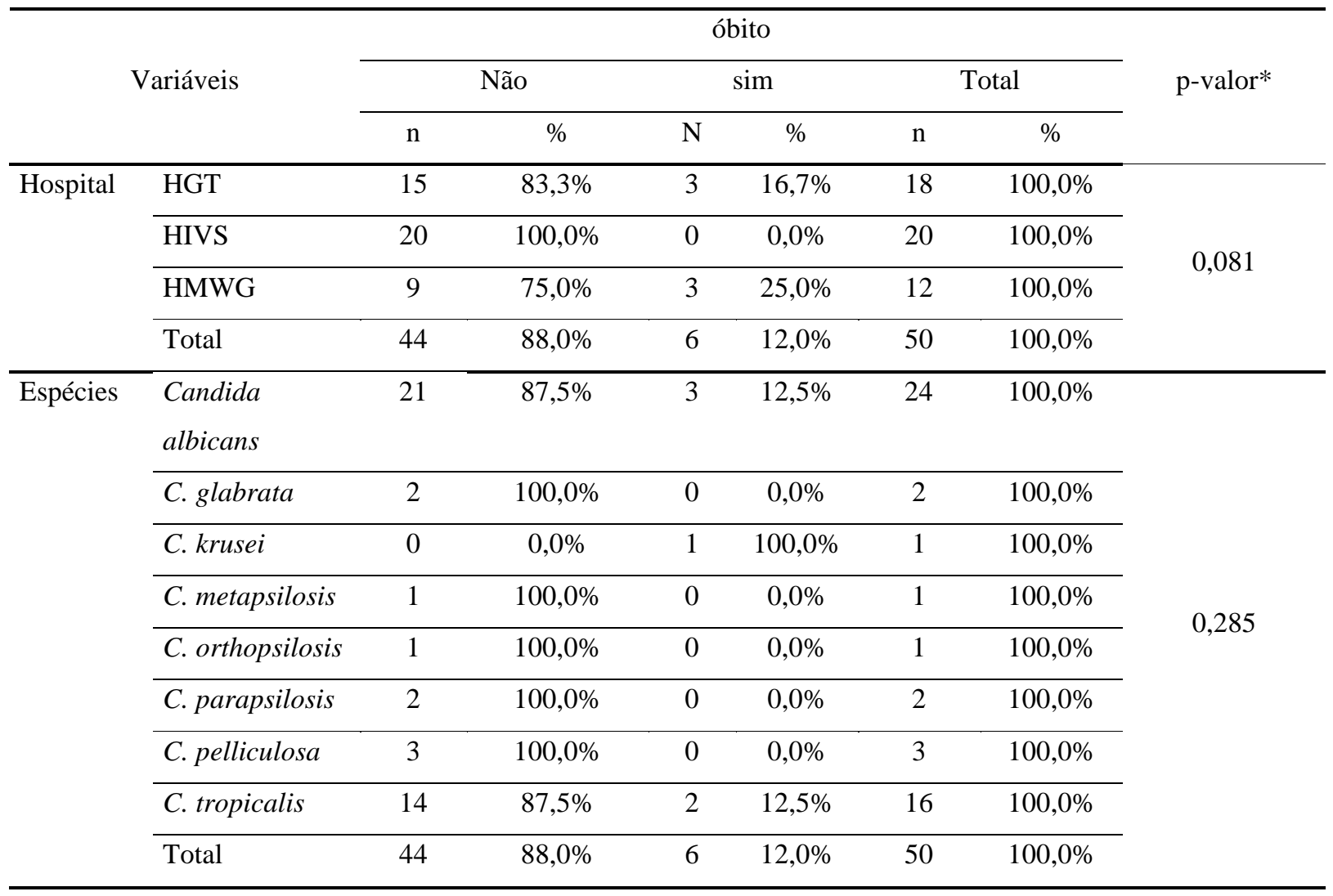

*teste qui-quadrado

Fonte: Autores.

Conforme tabela abaixo, não houve diferença significante na distribuição dos óbitos quando se compara casos nos diferentes hospitais (p-valor: 0,081) e também quando se compara a espécies de Candida (p-valor: 0,285). Em relação à sobrevida para óbito quando comparado às espécies $C$. tropicalis e $C$. albicans, mais prevalentes entre os pacientes nos três hospitais, o percentual de mortes foi de 12,5\% para ambos os pacientes. (Tabela 2). Ainda, apesar de os pacientes acometidos por C. tropicalis possuírem uma média de sobrevivência levemente maior que $C$. albicans, a hipótese obtida pelo teste long rank considera similar a distribuição da sobrevivência entre os pacientes que contraíram infecção, tanto por C. albicans quanto por C. tropicalis (41,688 vs. 39,189, respectivamente), ou seja, o tempo de óbito ocorre em prazos semelhantes para ambas (p-valor: 0,863), como observado no Gráfico 1.

Em relação à sobrevida para óbito quando comparado pacientes dos hospitais $\mathrm{HB}$ e HC, o percentual de mortes é de 16,67\% no HB e 25\% no HC. O hospital HA não tem dados de óbito para os pacientes participantes do estudo. Quando aplicado o teste long rank, foi possível perceber que há semelhança sutil na distribuição da sobrevivência entre pacientes dos hospitais HB e HC, ou seja, o tempo de óbito ocorre em prazos semelhantes para ambos (p-valor: 0,710). Ainda, conforme apresentado na Tabela 3, os pacientes que contraíram infecções por $C$. tropicalis possuem uma média de sobrevivência maior, porém bem próxima da média dos pacientes que contraíram C. albicans (41,688 vs. 39,189, respectivamente). 
Gráfico1: O gráfico de sobrevivência espelha os dados permitindo verificar quais das curvas apresenta melhor resultado, quanto mais acima está a curva, menos mortes. Dessa forma, pode-se observar que as curvas das duas espécies são bem próximas, justificando o resultado de que não há diferença significativa na média de sobrevivência entre elas.

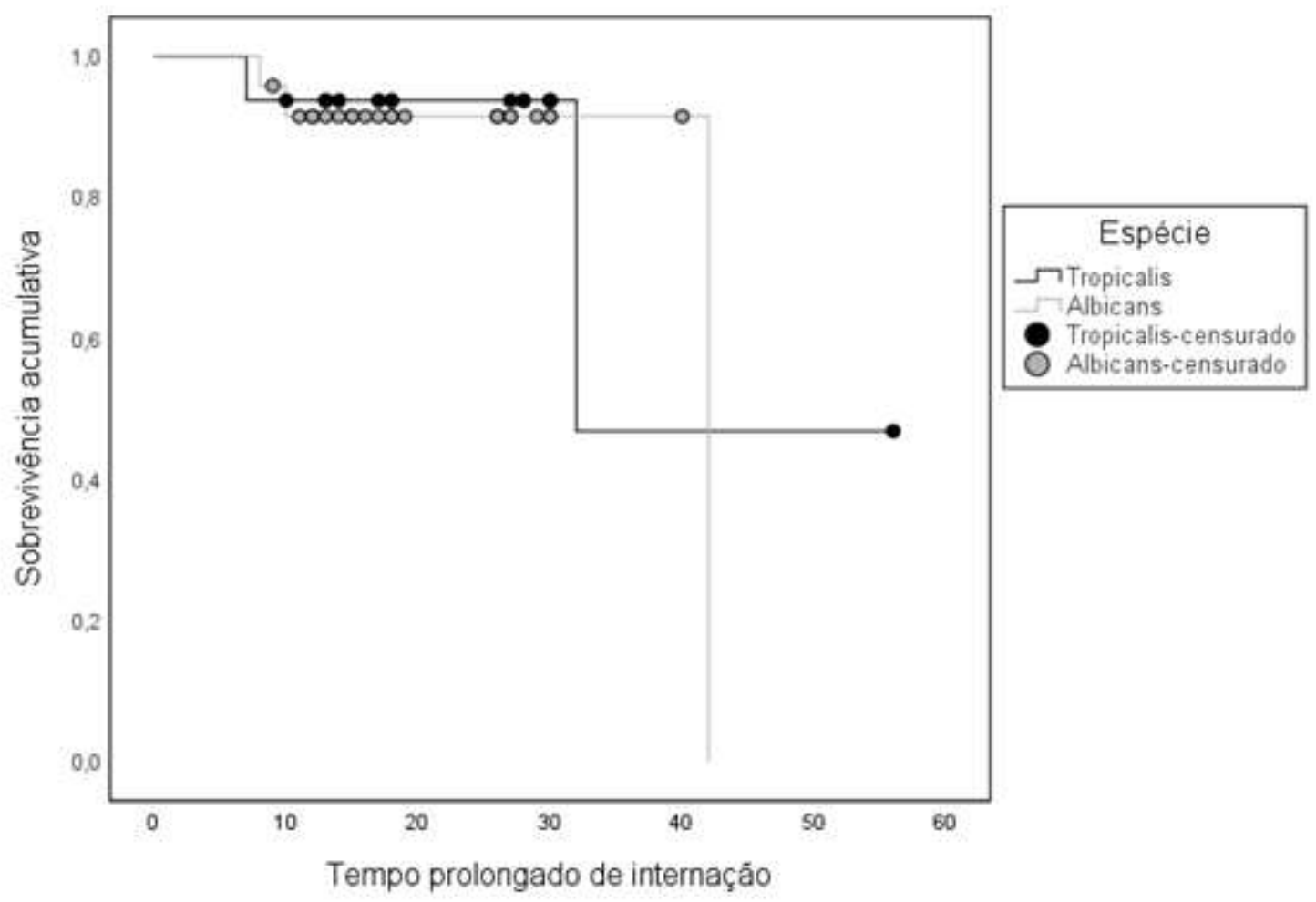

Fonte: Autores.

O gráfico de sobrevivência espelha os dados permitindo verificar quais das curvas apresenta melhor resultado, quanto mais acima está a curva, menos mortes.

$\mathrm{Na}$ Tabela 4 são descritos os valores de concentração inibitória mínima (CIM) dos antifúngicos, anfotericina B, fluconazol, voriconazol, micafungina, caspofungina e anidulafungina, testados frente a todas as 50 amostras, bem como para as cepas ATCC (controle de qualidade). Dos 24 isolados de C. albicans, observou-se resistência a anfotericina B (1/4\%), anidulafungina (1/4\%), micafungina (1/4\%) e voriconazol (1/4\%), 3 (11\%) sensíveis dose dependente a voriconazol e 1 (4\%) a fluconazol. Das 16 amostras de C. tropicalis, 1 (6\%) isolado foi resistente à anfotericina B e $1(6 \%)$ a voriconazol, enquanto 2 (11\%) foram sensível dose dependente a fluconazol e $2(11 \%)$ a voriconazol. Dentre as 3 cepas de $C$. pelliculosa, 1 (25\%) foi resistente e 1 (25\%) sensível dose dependente ao voriconazol. Enquanto que das duas amostras de C. parapsilosis $1(50 \%)$ foi resistente à anidulafungina e o único isolado de $C$. krusei foi resistente ao mesmo antifúngico. 
Tabela 4: concentração inibitória mínima (MIC) dos antifúngicos.

\section{Concentração Inibitória Mínima-CIM $(\mu \mathrm{g} / \mathrm{mL})$}

\begin{tabular}{|c|c|c|c|c|c|c|c|}
\hline \multirow[t]{2}{*}{ Espécie } & \multirow[t]{2}{*}{ Isolados } & \multirow{2}{*}{$\frac{\text { Anfotericina B }}{48 \mathrm{~h}}$} & \multirow{2}{*}{$\frac{\text { Fluconazol }}{24 \mathrm{~h}}$} & \multirow{2}{*}{$\frac{\text { Vorinazol }}{24 \mathrm{~h}}$} & \multirow{2}{*}{$\frac{\text { Micafugina }}{24 \mathrm{~h}}$} & \multirow{2}{*}{$\begin{array}{l}\text { Caspofugina } \\
24 \mathrm{~h}\end{array}$} & \multirow{2}{*}{$\begin{array}{l}\text { Anidulafugina } \\
24 \mathrm{~h}\end{array}$} \\
\hline & & & & & & & \\
\hline \multirow{24}{*}{$\begin{array}{l}\text { Candida } \\
\text { albicans }\end{array}$} & CRN1 & $1(\mathrm{~S})$ & $8(\mathrm{R})$ & $2(\mathrm{R})$ & $0,5(\mathrm{~S})$ & $0,25(\mathrm{~S})$ & $4(\mathrm{R})$ \\
\hline & CRN3 & $8(\mathrm{R})$ & $64(\mathrm{R})$ & $2(\mathrm{R})$ & $1(\mathrm{R})$ & $0,5(\mathrm{SDD})$ & $1(\mathrm{R})$ \\
\hline & CRN7 & $0,06(\mathrm{~S})$ & $0,125(\mathrm{~S})$ & $0,06(\mathrm{~S})$ & $4(\mathrm{R})$ & $0,25(\mathrm{~S})$ & $0,25(\mathrm{~S})$ \\
\hline & CRN12 & $1(\mathrm{~S})$ & $0,25(\mathrm{~S})$ & $0,5(\mathrm{SDD})$ & $2(\mathrm{R})$ & $0,03(\mathrm{~S})$ & $0,125(\mathrm{~S})$ \\
\hline & CRN13 & $0,06(S)$ & $0,125(\mathrm{~S})$ & $0,06(S)$ & $0,06(\mathrm{~S})$ & $0,06(\mathrm{~S})$ & $2(\mathrm{R})$ \\
\hline & CRN14 & $0,06(\mathrm{~S})$ & $0,125(\mathrm{~S})$ & $0,06(\mathrm{~S})$ & $0,06(\mathrm{~S})$ & $0,5(\mathrm{SDD})$ & $2(\mathrm{R})$ \\
\hline & CRN15 & $0,25(\mathrm{~S})$ & $0,125(\mathrm{~S})$ & $0,06(\mathrm{~S})$ & $0,06(\mathrm{~S})$ & $0,5(\mathrm{SDD})$ & $0,5(\mathrm{SDD})$ \\
\hline & CRN16 & $0,06(\mathrm{~S})$ & $0,125(\mathrm{~S})$ & $0,125(\mathrm{~S})$ & $2(\mathrm{R})$ & $0,5(\mathrm{SDD})$ & 0,5(SDD) \\
\hline & CRN17 & $1(\mathrm{~S})$ & $1(\mathrm{~S})$ & $1(\mathrm{R})$ & $0,25(\mathrm{~S})$ & $0,5(\mathrm{SDD})$ & $0,25(\mathrm{~S})$ \\
\hline & CRN18 & $1(\mathrm{~S})$ & $0,25(\mathrm{~S})$ & $8(\mathrm{R})$ & $0,125(\mathrm{~S})$ & $1(\mathrm{R})$ & $1(\mathrm{R})$ \\
\hline & CRN19 & $0,06(\mathrm{~S})$ & $0,5(\mathrm{~S})$ & $0,125(\mathrm{~S})$ & $1(\mathrm{R})$ & 0,5(SDD) & $0,5(\mathrm{SDD})$ \\
\hline & CRN22 & $0,03(\mathrm{~S})$ & $0,0125(\mathrm{~S})$ & $0,03(\mathrm{~S})$ & $0,25(\mathrm{~S})$ & $0,06(\mathrm{~S})$ & $1(\mathrm{R})$ \\
\hline & CRN27 & $0,03(\mathrm{~S})$ & $0,25(\mathrm{~S})$ & $0,03(\mathrm{~S})$ & $0,25(\mathrm{~S})$ & $0,06(S)$ & $0,06(\mathrm{~S})$ \\
\hline & CRN30 & $0,03(\mathrm{~S})$ & $0,25(\mathrm{~S})$ & $0,25(\mathrm{SDD})$ & $0,06(\mathrm{~S})$ & $0,25(\mathrm{~S})$ & $0,125(\mathrm{~S})$ \\
\hline & CRN32 & $0,03(\mathrm{~S})$ & $0,125(\mathrm{~S})$ & $0,03(\mathrm{~S})$ & $0,06(\mathrm{~S})$ & $0,25(\mathrm{~S})$ & $0,5(\mathrm{SDD})$ \\
\hline & CRN34 & $0,125(\mathrm{~S})$ & $0,25(\mathrm{~S})$ & $0,03(\mathrm{~S})$ & $0,06(\mathrm{~S})$ & $0,03(\mathrm{~S})$ & $2(\mathrm{R})$ \\
\hline & CRN40 & $1(\mathrm{~S})$ & $0,5(\mathrm{~S})$ & $0,06(\mathrm{~S})$ & $0,125(\mathrm{~S})$ & $0,06(\mathrm{~S})$ & $0,125(\mathrm{~S})$ \\
\hline & CRN41 & $1(\mathrm{~S})$ & 4(SDD) & $0,06(\mathrm{~S})$ & $0,125(\mathrm{~S})$ & $0,03(\mathrm{~S})$ & $2(\mathrm{R})$ \\
\hline & CRN42 & $0,25(\mathrm{~S})$ & $0,5(\mathrm{~S})$ & $0,06(\mathrm{~S})$ & $0.06(\mathrm{~S})$ & $0,06(\mathrm{~S})$ & $0,5(\mathrm{SDD})$ \\
\hline & CRN43 & $0,125(\mathrm{~S})$ & $0,25(\mathrm{~S})$ & $0,06(\mathrm{~S})$ & $1(\mathrm{R})$ & $0,25(\mathrm{~S})$ & $0,5(\mathrm{SDD})$ \\
\hline & CRN44 & $0,03(\mathrm{~S})$ & $0,125(\mathrm{~S})$ & $0,06(\mathrm{~S})$ & $1(\mathrm{R})$ & $0,03(\mathrm{~S})$ & $0,25(\mathrm{~S})$ \\
\hline & CRN47 & $0,03(\mathrm{~S})$ & $32(\mathrm{R})$ & $8(\mathrm{R})$ & $0,125(\mathrm{~S})$ & $0,25(\mathrm{~S})$ & $0,5(\mathrm{SDD})$ \\
\hline & CRN49 & $0,25(\mathrm{~S})$ & $1(\mathrm{~S})$ & $0,125(\mathrm{~S})$ & $2(\mathrm{R})$ & $0,125(\mathrm{~S})$ & $1(\mathrm{R})$ \\
\hline & CRN50 & $0,03(\mathrm{~S})$ & $0,5(\mathrm{~S})$ & $0,125(\mathrm{~S})$ & $4(\mathrm{R})$ & $0,5(\mathrm{SDD})$ & $0,125(\mathrm{~S})$ \\
\hline \multirow[t]{5}{*}{ C. tropicalis } & CRN2 & $0,5(\mathrm{~S})$ & $0,25(\mathrm{~S})$ & $1(\mathrm{R})$ & $0,5(\mathrm{SDD})$ & $0,125(\mathrm{~S})$ & $8(\mathrm{R})$ \\
\hline & CRN4 & $2(\mathrm{R})$ & $0,25(\mathrm{~S})$ & $8(\mathrm{R})$ & $0,125(\mathrm{~S})$ & $2(\mathrm{R})$ & $0,25(\mathrm{~S})$ \\
\hline & CRN6 & $0,5(\mathrm{~S})$ & $0,125(\mathrm{~S})$ & $2(\mathrm{R})$ & $0,03(\mathrm{~S})$ & $0,5(\mathrm{SDD})$ & $0,06(\mathrm{~S})$ \\
\hline & CRN8 & $0,5(\mathrm{~S})$ & $0,25(\mathrm{~S})$ & $1(\mathrm{R})$ & $0,5(\mathrm{SDD})$ & $0,03(\mathrm{~S})$ & $0,25(\mathrm{~S})$ \\
\hline & CRN10 & $1(\mathrm{~S})$ & $0,125(\mathrm{~S})$ & $0,06(\mathrm{~S})$ & $0,125(\mathrm{~S})$ & $0,5(\mathrm{SDD})$ & $0,03(\mathrm{~S})$ \\
\hline
\end{tabular}




\begin{tabular}{|c|c|c|c|c|c|c|c|}
\hline & CRN20 & $0,03(\mathrm{~S})$ & $0,25(\mathrm{~S})$ & $0,06(\mathrm{~S})$ & $0,06(\mathrm{~S})$ & $2(\mathrm{R})$ & $2(\mathrm{R})$ \\
\hline & CRN21 & $0,03(\mathrm{~S})$ & $0,125(\mathrm{~S})$ & $0,03(\mathrm{~S})$ & $2(\mathrm{R})$ & $0,03(\mathrm{~S})$ & $2(\mathrm{R})$ \\
\hline & CRN23 & $0,03(\mathrm{~S})$ & $32(\mathrm{R})$ & $0,06(\mathrm{~S})$ & $0,25(\mathrm{~S})$ & $2(\mathrm{R})$ & $2(\mathrm{R})$ \\
\hline & CRN26 & $0,03(\mathrm{~S})$ & $0,5(\mathrm{~S})$ & $0,125(\mathrm{~S})$ & $0,03(\mathrm{~S})$ & $4(\mathrm{R})$ & $0,06(\mathrm{~S})$ \\
\hline & CRN28 & $0,06(\mathrm{~S})$ & $0,5(\mathrm{~S})$ & $0,06(\mathrm{~S})$ & $0,5(\mathrm{SDD})$ & $1(\mathrm{R})$ & $0,03(\mathrm{~S})$ \\
\hline & CRN29 & $0,03(\mathrm{~S})$ & $0,5(\mathrm{~S})$ & $0,06(\mathrm{~S})$ & $0,125(\mathrm{~S})$ & $0,06(\mathrm{~S})$ & $0,03(\mathrm{~S})$ \\
\hline & CRN31 & $0,25(\mathrm{~S})$ & $0,125(\mathrm{~S})$ & $0,125(\mathrm{~S})$ & $0,25(\mathrm{~S})$ & $0,125(\mathrm{~S})$ & $0,125(\mathrm{~S})$ \\
\hline & CRN39 & $0,03(\mathrm{~S})$ & 4(SDD) & $0,25(\mathrm{SDD})$ & $0,25(\mathrm{~S})$ & $2(\mathrm{R})$ & $1(\mathrm{R})$ \\
\hline & CRN36 & $0,06(\mathrm{~S})$ & $1(\mathrm{~S})$ & $0,125(\mathrm{~S})$ & $0.25(\mathrm{~S})$ & $0,125(\mathrm{~S})$ & $0,5(\mathrm{SDD})$ \\
\hline & CRN45 & $0,03(\mathrm{~S})$ & $0,5(\mathrm{~S})$ & $0,125(\mathrm{~S})$ & $0,06(\mathrm{~S})$ & $0,03(\mathrm{~S})$ & $0,5(\mathrm{SDD})$ \\
\hline & CRN46 & $0,25(\mathrm{~S})$ & $32(\mathrm{R})$ & $8(\mathrm{R})$ & $0,125(\mathrm{~S})$ & $0,03(\mathrm{~S})$ & $0,5(\mathrm{SDD})$ \\
\hline \multirow[t]{3}{*}{ C. pelliculosa } & CRN5 & $1(\mathrm{~S})$ & $1(\mathrm{~S})$ & 2(SDD) & $0,06(\mathrm{~S})$ & $0,25(\mathrm{~S})$ & $1(\mathrm{~S})$ \\
\hline & CRN9 & $0,5(\mathrm{~S})$ & $0,5(\mathrm{~S})$ & $4(\mathrm{R})$ & $1(\mathrm{~S})$ & $0,6(S)$ & $0,25(\mathrm{~S})$ \\
\hline & CRN11 & $1(\mathrm{~S})$ & $1(\mathrm{~S})$ & 2(SDD) & $2(\mathrm{~S})$ & $1(\mathrm{R})$ & $0,03(\mathrm{~S})$ \\
\hline \multirow[t]{2}{*}{ C. glabrata } & CRN24 & $0,03(\mathrm{~S})$ & $0,25(\mathrm{~S})$ & $0,125(\mathrm{~S})$ & $0,25(\mathrm{R})$ & $0,06(\mathrm{~S})$ & $0,06(\mathrm{~S})$ \\
\hline & CRN25 & $0,06(\mathrm{~S})$ & $0,25(\mathrm{~S})$ & $0,03(\mathrm{~S})$ & $0,125(\mathrm{SDD})$ & $0,125(\mathrm{~S})$ & $0,125(\mathrm{~S})$ \\
\hline $\begin{array}{l}\text { C. } \\
\text { orthopsilosis }\end{array}$ & CRN33 & $0,03(\mathrm{~S})$ & $0,25(\mathrm{~S})$ & 0,25 (SDD) & $0,03(\mathrm{~S})$ & $0,125(\mathrm{~S})$ & $1(\mathrm{~S})$ \\
\hline \multirow{2}{*}{$\begin{array}{l}\text { C. } \\
\text { parapsilosis }\end{array}$} & CRN35 & $0,03(\mathrm{~S})$ & $1(\mathrm{~S})$ & $0,25(\mathrm{SDD})$ & $2(\mathrm{~S})$ & $0,03(\mathrm{~S})$ & $0,03(\mathrm{~S})$ \\
\hline & CRN37 & $0,06(\mathrm{~S})$ & $0,5(\mathrm{~S})$ & $0,125(\mathrm{~S})$ & $0,5(\mathrm{~S})$ & $0,03(\mathrm{~S})$ & $16(\mathrm{R})$ \\
\hline $\begin{array}{l}\text { C. } \\
\text { metapsilosis }\end{array}$ & CRN38 & $0,03(\mathrm{~S})$ & $0,25(\mathrm{~S})$ & $0,125(\mathrm{~S})$ & $0.25(\mathrm{~S})$ & $0,25(\mathrm{~S})$ & $0,5(\mathrm{~S})$ \\
\hline C. krusei & CRN48 & $0,25(\mathrm{~S})$ & 0,25 & $0,06(\mathrm{~S})$ & $0,25(\mathrm{~S})$ & $1(\mathrm{R})$ & $4(\mathrm{R})$ \\
\hline $\begin{array}{l}\text { C. } \\
\text { parapsilosis }\end{array}$ & $\begin{array}{l}\text { ATCC22 } \\
019\end{array}$ & 1 & 4 & 0,5 & 1 & 0,25 & 0,5 \\
\hline C. krusei & $\begin{array}{l}\text { ATCC62 } \\
58\end{array}$ & 1 & 4 & 0,125 & 0,25 & 0,125 & 0,5 \\
\hline MIC50 & & 0,06 & 0,25 & 0,125 & 0,25 & 0,25 & 0,5 \\
\hline MIC90 & & 1 & 4 & 2 & 2 & 1 & 2 \\
\hline
\end{tabular}

S=Sensível, SDD= Suscetível Dose Dependente, $\mathrm{R}=$ Resistente.

Fonte: Autores.

\section{Discussão}

Acometendo hoje mais de 250.000 pessoas por ano, a candidemia tornou-se um problema de saúde pública mundial, sem distinção de sexo, afetando pessoas de todas as faixas etárias (Pappas et al. 2018, Pinhati et al. 2016). Nesse âmbito, no estudo não foi observado diferença na incidência de candidemia com relação ao sexo, enquanto que os indivídus mais acometidos 
pela candidemia foram pacientes jovens. Estudos anteriores têm relatado que esta população apresenta maior risco para desenvolver candidemia devido às terapias mais agressivas, necessitando de estratégias de intervenções apropriadas para redução da morbidade e mortalidade (Caggiano et al. 2017, Chakrabarti et al. 2020).

Dentre os agentes etiológicos, C. albicans foi a espécie mais comum causando candidemia, seguida por C. tropicalis. Ainda, durante o período de realização do estudo foi notado o aparecimento de outras espécies de importância clínica como $C$. pelliculosa, C. parapsilosis e C. glabrata. Existem hoje, mais de 17 espécies diferentes de Candida identificadas como patógenos causadores de alterações da corrente sanguínea (BSIs). Embora C. albicans ainda seja considerada o patógeno dominante da candidemia, o aumento das taxas de infecções invasivas por espécies de Candida não-albicans e resistência a antifúngicos em diferentes partes do mundo, tem preocupado as instituições de saúde (Liu et al. 2019, Pinhati et al. 2016, Doi et al. 2016, Santos et al. 2018). Assim, esse panorama é semelhante ao observado por outros estudos, onde mais de $90 \%$ das candidemias são causadas por C. albicans, C. tropicalis, C. parapsilosis, C. glabrata e C. krusei (Xiao et al. 2019, Santos et al. 2018). Desta forma, uma compreensão da epidemiologia da candidemia e da suscetibilidade antifúngica das espécies, é imprescindível para orientar a vigilância nas estratégias de tratamento e controle destas infecções através do diagnóstico precoce e prevenção.

A maioria dos pacientes em nosso estudo, tiveram longos períodos de cateterismo do trato urinário, nutrição parenteral, cateterismo venoso central, uso de corticoides e imunossupressão. Segundo Tadec et al. (2016) esses fatores se mostram responsáveis pelas infecções fúngicas. Associado a esses riscos, são mais susceptíveis a desenvolverem infecções relacionadas à Candida, pacientes com neoplasias hematológicas, neutropenia, em uso de ventilação mecânica e aqueles em tratamento antibacterianos prolongados e de largo espectro em UTI (Fortún; Goia, 2017; Peixoto et al. 2014). Observamos que apesar dos avanços clínicos no suporte de paciente crítico, a candidemia ainda é de difícil diagnóstico, resultando em internação prolongada e aumento dos casos de mortalidade.

Em se tratando da mortalidade ocasionada pelas espécies de Candida, foram observados seis óbitos causados por Candida albicans, $C$. tropicalis e C. krusei, o que representa um percentual de $12 \%$ dos casos de candidemia deste estudo. De maneira similar, outros estudos têm demonstrado que a candidemia é uma condição preocupante, sobretudo, devido a sua alta taxa de mortalidade (Magill et al., 2014; Kullberg, et al. 2015). Skrobik e colaboradores (2013) observaram, ainda, que falhas no diagnóstico podem conduzir ao atraso na terapêutica antifúngica e aumento considerável na taxa de mortalidade. Demonstrando a importância do prévio diagnóstico das diferentes espécies de Candida causadoras de candidemia, associado ao conhecimento do padrão de susceptibilidade aos antifúngicos.

Outro dado relevante é que a sobrevida dos pacientes infectados por C. albicans e C. tropicalis é estatisticamente semelhante com tempo de internação hospitalar prolongado até a morte do paciente, o que leva a crer que essas espécies possuem a mesma relevância quanto ao seu potencial patogênico. Similar ao aqui observado, Li e colaboradores (2017) perceberam que a mortalidade (30\%) ocasionada pela candidemia (média de 30 dias) foi significativamente maior quando comparada com a mortalidade das infecções bacterianas. Esses dados apontam o alto impacto negativo para o sistema de saúde ocasionado pela candidemia, o que requer uma abordagem de diagnóstico e tratamento robusto a fim de melhorar esse panorama grave.

A maioria dos isolados de Candida (>80\%) foram sensíveis ou suscetíveis dose-dependente a anfotericina B, fluconazol, voriconazol e as equinocandinas. Embora a anfotericina B tenha uma ação rápida contra a maioria das cepas de Candida e a resistência geralmente considerada rara (Kucukates et al. 2016), em nosso estudo encontramos cepas de $C$. albicans e $C$. tropicalis resistentes a este fármaco. Observamos resistência de isolados de C. parapsilosis e C. krusei a anidulafungina, embora seja raro esta apresentação antifúngica para as equinocandinas (Maubon et al. 2014, Matta et al. 2017).

O reconhecimento rápido da candidemia e o início imediato da terapia antifúngica apropriada é um fator determinante do resultado. As atuais diretrizes recomendam o tratamento antifúngico empírico, embora muitas vezes falhe em conferir 
qualquer benefício aos pacientes. Para certas espécies, a suscetibilidade a agentes antifúngicos pode ser prevista com base em dados de suscetibilidade epidemiológica (Xiao et al. 2019, Deconinck et al. 2016).

\section{Conclusão}

A espécie prevalente no estudo foi C. albicans, sendo essa o principal agente responsável pela mortalidade. A idade juvenil, antibioticoterapia de largo espectro, imunossupressão, doenças de base preexistentes e neutropenia se destacaram como as principais condições predisponentes ao surgimento da candidemia na UTI. Desta forma, especial atenção deve ser direcionada aqueles pacientes que apresentarem estes fatores de risco a fim de realizar uma terapia profilática prévia. Apesar de não termos observado a relação entre o uso de dispositivos médicos com o surgimento da candidemia, é necessário melhor atenção das equipes médicas a essa questão, tendo em vista que esses dispositivos são comumente relatados em outros estudos como importantes fatores de risco para esta grave infecção.

\section{Apoio financeiro}

\section{CAPES, CNPQ, FACEPE}

\section{Referências}

Andersen, K. M., Kristoffersen, A. K., Ingebretsen, A., Vikholt, K. J., Ortengren, U. T., Olsen, I., Enersen, M., \& Gaustad. P. (2016). Diversity and antifungal susceptibility of Norwegian Candida glabrata clinical isolates. Journal of Oral Microbiology, 8(10):3402. http://dx.doi.org/10.3402/jom.v8.29849

Caggiano, G., Lovero, G., Giglio, O., Barbuti, G., Montagna, O., Laforgia, N., \& Montagna, M. T. (2017). Candidemia in the Neonatal Intensive Care Unit: A Retrospective, Observational Survey and Analysis of Literature Data. BioMed Research International, 2017:7901763. 10.1155 / 2017/7901763

Chakrabarti, A., Sood, P., Rudramurthy, S. M., Chen, S., Jillwin, J., Iyer, J., Sharma, A., Harish, B. N., Roy, I., Kindo, A. J., Chhina, D., Savio, J., Mendiratta, D., Capoor, M. R., Das, S., Arora, A., Chander, J., Xess, I., Boppe, A., Ray, U., Rao, R., Eshwara, V. K., Joshi, S., Pate, A., Sardana, R., Shetty, A., \& Pamidimukkala, U. (2020). Characteristics, outcome and risk factors for mortality of paediatric patients with ICU-acquired candidemia in India: A multicentre prospective study. Mycoses, 00:1-15. 10.3390/jof3030041

Clinical and Laboratory Standards Institute (CLSI) (2008). Reference method for broth dilution testing of yeasts: Approved standardthird edition M27-A3. Wayne, PA: Clinical Laboratory Standards Institute. https://www.clsi.org/media/1461/m27a3_sample.pdf

Clinical and Laboratory Standards Institute (CLSI) (2012). Reference method for broth dilution antifungal susceptibility testing of yeasts; fourth informational supplement. Wayne: Clinical and Laboratory Standards Institute. (Document M27-S4).

Deconinck, L., Meybeck, A., Pradier, M., Patoz, P., Melliez, H., \& Senneville, E. (2016). Community acquired fungemia caused by Candida pulcherrima: diagnostic contribution of MALDI-TOF mass spectrometry. Annals of Clinical Microbiology and Antimicrobials, 15:14. 10.1186/s12941-016-0129-1

Doi, A. M., Pignatari, A. C. C., Edmond, M., Marra, A. R., Camargo, L. F. A., Siqueira, R. A., Mota, V. P., \& Colombo, A. L. (2016). Epidemiology and Microbiologic Characterization of Nosocomial Candidemia from a Brazilian National Surveillance Program. Journal Plos One, 11:e0146909. 10.1371/journal.pone.0146909

Fortún, J., \& Gioia, F. (2017). Invasive Candidiasis in the neutropenic patient. Revista Especialista em Quimioterapia, 30(Suppl. 1):22-25.

Ghosh, A. K., Paul, S., Sood, P., Rudramurthy, S. M., Rajbanshi, A., Jillwin, T. J., \& Chakrabarti, A. (2015). Matrix-assisted laser desorption ionization time-of-flight mass spectrometry for the rapid identification of yeasts causing bloodstream infections. Clinical Microbiology and Infection, 21:372-378. 10.1016 / j.cmi.2014.11.009

Inácio, C. P, Rocha, A. P., Barbosa, R. N., Oliveira, N. T., Silva, J. C., Lima-Neto, R. G., Macedo, D. P. C., \& Neves, R. P. (2016). Experimental white piedra: a robust approach to ultrastructural analysis, scanning electron microscopy and etiological discoveries. Experimental Dermatology, 25(1), 79-81.

Kaur, H., \& Chakrabarti, A. (2017). Strategies to Reduce Mortality in Adult and Neonatal Candidemia in Developing Countries. Journal of Fungi, 3(3):41. ho de 2017. $10.3390 /$ jof3030041

Kucukates, E., Gultekin, N. N., Alisan, Z., Hondur, N., \& Ozturk, R. (2016). Identification of Candida species and susceptibility testing with Sensititre YeastOne microdilution panel to 9 antifungal agentes. Saudi Medical Journal, 37(7):750-757. 10.15537 / smj.2016.7.13412

Kullberg, B. J., \& Arendrup, M. C. (2015). Invasive Candidiasis. N Engl J Med, 373:1445-56. 10.1056/NEJMra1315399

Li, D., Xia, R., Zhang, Q., Bai, C., Li, Z., \& Zhang, P. (2017). Evaluation of candidemia in epidemiology and risk factors among cancer patients in a cancer center of China: an 8-year case-control study. BMC Infectectious Diseases, 17:536. 10.1186/s12879-017-2636-x

Liu, W., Lai, C., Li, M., Wu, C., Ko, W., Hung, Y., Tang, H., \& Hsueh, P. (2019). Clinical manifestations of candidemia caused by uncommon Candida species and antifungal susceptibility of the isolates in a regional hospital in Taiwan, 2007 e 2014. Journal of Microbiology, Immunology and Infection, 52;612-619. 
Lovero, G., Gilio, O., Montagna, O., Diella, G., Divenuto, F., Lopuzzo, M., Rutigliano, S., Laforgia, N., Caggiano, G., \& Montagna, M.T. (2016). Epidemiologia da candidemia em unidades de terapia intensiva neonatal: um problema persistente de saúde pública. Ann Ig, 28:282-287. 10.7416/ai.2016.2107

Magill, S. S., Edwards, J. R., Bamberg, W., Beldavs, Z. G., Dumyati, G., Kainer, M. A., Lynfield, R., Maloney McAllister-Hollod, L., Nadle, J., Ray, S. M., Thompson, D. L., Wilson, L. E., \& Fridkin, S. K. (2014). Multistate point-prevalence survey of health care-associated infections. N Engl J Med., 370:1198-208. 10.1056 / NEJMoa1306801

Matta, D. A., Souza, A. C. R., \& Colombo, A. L. (2017). Revisiting Species Distribution and Antifungal Susceptibility of Candida Bloodstream Isolates from Latin American Medical Centers. Journal of Fungi, 3(2):24. 10.3390 / jof3020024

Maubon, D., Garnaud, C., Calandra, T., Sanglard, D., \& Cornet, M. (2014). Resistance of Candida spp. to antifungal drugs in the ICU: where are we now? Intensive Care Medicine, 40(9):1241-55. 10.1007 / s00134-014-3404-7

Merseguel, K. B., Nishikaku, A. S., Rodrigues, A. M., Padovan, A. C., Ferreira, R. C., Melo, A. S. A., Briones, M. R., \& Colombo, A. L. (2015). Genetic diversity of medically important and emerging Candida species causing invasive infection. BMC Infectious Diseases, 15:57. 10.1186 / s12879-015-0793-3

Pappas, P. G., Lionakis, M. S., Arendrup, M. C., Ostrosky-Zeichner, L., \& Kullberg, B. J. (2018). Candidíase invasiva. Nat Rev Dis Primers, 4:18026. $10.1038 /$ nrdp. 2018.26

Peixoto, J. V., Rocha, M. V., Nascimento, R. T. L., Moreira, V. L., \& Kashiwabara, T. G. B. (2014). Candidíase: uma revisão de literatura. Brazilian Journal of Surgery and Clinical Research, 8:75-82.

Pfaller, M. A., Wolk, D. M., \& Lowery, T. J. (2016). T2mr and t2candida: novel technology for the rapid diagnosis of candidemia and invasive candidiasis. Future microbiology, Future Medicine, 11(1):103-117. 10.2217 / fmb.15.111

Pinhati, H. M., Casulari, L. A., Souza, A. C., Siqueira, R. A., Damasceno, C. M., \& Colombo, A. L. (2016). Outbreak of candidemia caused by fluconazole resistant Candida parapsilosis strains in an intensive care unit. BMC Infectious Disease, 16:433. 10.1186/s12879-016-1767-9

Santos, G. C. O., Vasconcelos, C. C., Lopes, A. J. O., Cartágenes, M. S. S., Filho, A. K. D. B., Nascimento, F. R. F., Ramos, R. M., Pires, E. R. R. B., Andrade, M. S., Rocha, F. M. G., \& Monteiro, C. A. (2018). Infecções por Candida e estratégias terapêuticas: mecanismos de ação para agentes tradicionais e alternativos. Frontiers in Microbiology, 9:1351. 10.3389/fmicb.2018.01351

Skrobik, Y., \& Laverdiere, M. (2013). Why Candida sepsis should matter to ICU physicians. Crit Care Clin. 2013; 29:853-64. 10.1016 / j.ccc.2013.06.007

Tadec, L., Talarmin, J., Gastinne, T., Bretonnière, C., Miegeville, M., Le Pape, P., \& Morio, F. (2016). Epidemiology, risk factor, species distribution, antifungal resistance and outcome of Candidemia at a single French hospital: a 7-year study. Mycoses, 59:296-303. 10.1111/ myc.12470

White, T.J., Bruns, T., Lee, S., \& Taylor, J. (1999). Amplification and direct sequencing of fungal ribosomal RNA genes for phylogenetics. In PCR Protocols: A guide to Methods and Applications. San Diego, U.S.A.: Academic Press, 315-22. http://dx.doi.org/10.1016/b978-0-12-372180-8.50042-1

Wu, P. F., Liu, W. L., Hsieh, M. H., Hii, I. M., Lee, Y. L., Lin, Y. T., Ho, M. W., Liu, C. E., Chen, Y. H., \& Wang, F. D. (2017). Epidemiology and antifungal susceptibility of candidemia isolates of non-albicans Candida species from cancer patients. Emerging Microbes \& Infections, 11;6(10):e87. 10.1038/emi.2017.74

Xiao, Z., Wang, Q., Zhu, F., \& An, Y. (2019). Epidemiology, species distribution, antifungal susceptibility and mortality risk factors of candidemia among critically ill patients: a retrospective study from 2011 to 2017 in a teaching hospital in China. Antimicrobial Resistance and Infection Control, 8:89. https://doi.org/10.1186/s13756019-0534-2

Yapar, N. (2014). Epidemiology and risk factors for invasive candidiasis. Therapeutics and Clinical Risk Management, 10: 95-105. 10.2147 / TCRM.S40160 